\title{
Acupuncture in the Multimodal Biopsychosocial Pain Management. Towards a New Model in Clinical Practice
}

\author{
Agnès Mazic-de Sonis \\ Pain Clinic Chirec, Chirec, Site Parc Leopold, Brussels, Belgium \\ Email: agnes.mazicdesonis@chirec.be, painclinic.cpl@chirec.be
}

Received 29 May 2015; accepted 26 July 2015; published 30 July 2015

Copyright (C) 2015 by author and Scientific Research Publishing Inc.

This work is licensed under the Creative Commons Attribution-NonCommercial International License (CC

BY-NC).

http://creativecommons.org/licenses/by-nc/4.0/

(c) (i) (3) Open Access

\begin{abstract}
The last decennia the multidimensional nature of pain has been recognized and a multimodal biopsychosocial management has been proposed. Acupuncture has progressively gained a place in this multimodal approach. The principles of traditional Chinese medicine link three major themes, nutrition and microbiome, neuroplasticity, homeostasis and the side effects of medication. For patients with chronic pain and/or chronic pain refractory to conservative medicine it is important to assess all factors involved with the chronicity. The improved biological, genetic and epigenetic knowledge has contributed to a better understanding of the mechanism of action of acupuncture and integrative medicine. Moreover neuroimaging has been able to demonstrate the brain regions activated by acupuncture and also illustrates the differences with sham or placebo. Opponents often claim an absence or weakness of evidence. These claims are based on the observations that the initial reports are predominantly case reports and studies with a poor design quality. In the last decennia well-designed randomized trials have been published, thus improving the quality of the evidence. The most important findings are summarized in this article. As with all controlled trials the inclusion and exclusion criteria as well as the standardization of the treatment do not always reflect daily practice. Therefore we report patient cases as illustration of the integrative approach.
\end{abstract}

\section{Keywords}

Acupuncture, Multimodal Treatment, Nutrition, Chronobiology, Integrative Pain Medicine

\section{Introduction}

Since the second half of the previous century pain especially chronic pain has been recognized as a disease. 
Even for modern medicine chronic pain remains a complex, multi-facetal problem of nociception, inflammation and abnormal physiology in the nervous system. With the knowledge in epigenetics field environmental factors such as nutrition, and chronobiology can modify the parameters involved in all mechanisms of sensitization in chronic pain diseases.

This suggests the need for restoration of the hypersensitive structures to their normal status besides the analgesic treatment.

When Chinese reported performing surgery under acupuncture, indicative for an analgesic effect beyond the placebo effect the interest for Traditional Chinese Medicine (TCM) was raised in the Western world. Literature reviews suggested the possible value of acupuncture as adjuvant or complementary therapy for painful conditions.

Others found however no satisfactory prove of efficacy, mainly due to the fact that most of the studies on acupuncture up till 2000 were of low quality, showing all the weaknesses of most of the "interventional" pain management studies. Ernst et al. [1] performed a systematic review of the reviews on acupuncture and found 216 hits for the efficacy.

The understanding of the underlying mechanism of action has equally improved owing to the evolution of biology and neuroscience in animal and human research.

Animal studies have shown the integration of the central nervous system [2]. Neuroimaging such as Positron Emission Tomography (PET) and functional Magnetic Resonance Imaging (fMRI) demonstrated that acupuncture could modulate the activity in multiple cortical and subcortical brain areas [3]-[5].

The cerebral responses elicited by acupuncture stimulation are extensive; therefore it is difficult to determine the underlying mechanism regarding how does each brain region influence one another and how is the relationship among these regions.

The aim of this paper is to discuss the role of acupuncture in an integrated multimodal biopsychosocial pain management trajectory.

\subsection{Chronic Pain Affect Different Parameters}

A patient with chronic pain will attend the pain clinic with a pain symptom that is present for a prolonged period. Different factors that are equally influenced by the chronic pain problem should be evaluated during the patient assessment.

- Chronic pain and therapeutic failure

- Chronic pain and tolerance of the medication

- Chronic pain and sensitization

- The influence of the chronicity on the patient's general condition

- Which alternatives or complements can be envisioned, such as non-pharmacologic associations and pharmaconutrition?

- Which processes are activated when associating acupuncture?

The physiological revalidation and the clinical improvement can be explored with three main hypotheses:

- About the genetic disease, what is involved with the clinical expression?

- How can we improve the metabolism and effects of medical pain treatment?

- What process is activated by acupuncture treatment? Genetic and epigenetic factors combined, alter both gene expression and drug responses.

The progress made highlights the weaknesses in the knowledge. Future research should focus on what we don't know. We should study the natural history of disease, research non-drug based interventions, question diagnostic criteria, tighten the definition of competing interests, and research the actual long term benefits of drugs while promoting intellectual skepticism.

\subsection{Pain a Complex Phenomenon}

Traditional Chinese medicine (TCM), including acupuncture, has a long history of use for chronic and acute medical conditions, including pain .

TCM links three major themes, nutrition and microbiome, neuroplasticity, homeostasis and the side effects of medication. It cuts across all themes discussed here acupuncture, nutrition and the microbiome, neuroplasticity. TCM is a system of medicine that is ancient, complex, and detailed. It is holistic and views health as a result of 
balance and harmony between dynamic qualities in the relationship between different functions, rather than considering only structural, physical characteristics.

The status of a person depends for 30\% on genetic factors and $70 \%$ of epigenetic factors such as nutrition and intestinal flora, social, psychological and affective [6]. There are 20 genes affecting the sensibility for pain or that contribute to the variation in the response to pharmacologic treatment. Genetic and epigenetic factors combined modify the genetic expression of the pharmacologic response [7] (see Figure 1).

The epigenetic regulating system provides the cell with an ignition or blocking system for certain genes and is also sensitive to environmental factors [7].

The peripheral sensitization of the primary nociceptors also depends on the intra and extra cellular homeostasis. The bidirectional signalizing can be activated by the inflammation that it modulates. It was shown that even when there is no immunological response, certain bacterial infections could be accompanied by a hyperalgesic state [8] (see Figure 2).

There is a link between the peripheral and central nervous system. The connection geno-histo embryologic and neuro-somatic is the basis for the auricular representation that is used in auriculoacupuncture [9].

Ion channels play an essential role in the sensorial perception such as mechanical (touch), temperature (warm and cold) and pain. The group of Prof Lazdunski (CNRS) identified the molecular structure of the ion channels that convert a mechanical stimulation into an electrical signal. This group also identified a class of ion channels heavily expressed in the nociceptors. This are sensorial cells specialized in pain perception. Their activity is initiated by an extracellular acidosis. This type of ion channels reacts to an acidic stimulation and permits the generation of an electrical signal starting at the sensorial endings towards the spine and cerebral regions that integrate the pain sensation.

Other ion channels are responsible in the depolarizing phase of the action potential of the propagation of electric signals along the nerve fibers. Certain isoforms (Nav1.3, Nav1.7, Nav1.8 and Nav1.9) are preferentially, sometimes exclusively expressed in the peripheral nervous system. Local anesthetics, antiarythmics and anticonvulsants are sodium channel blockers of the sensory neurons. This means that sodium channels contribute to the hyperalgesia in case of neuropathic inflammation.

It is logical trying to produce treatments that target specifically the nature of the involved ion channels. The development of treatments that target those channels will result in drugs that have an increased therapeutic efficacy and are denuded of certain side effects [10].

The cellular membrane consists of proteins that allow or block the passage of information. This passage is facilitated by hydrophilic and polarized proteins that change forms with electrical loads that bind them in het environment or within the cell. Pharmacologic treatment can maintain a chronic state of malfunctioning.

The resultant neuro-inflammatory environment can cause activation of glial cells located in the central nervous

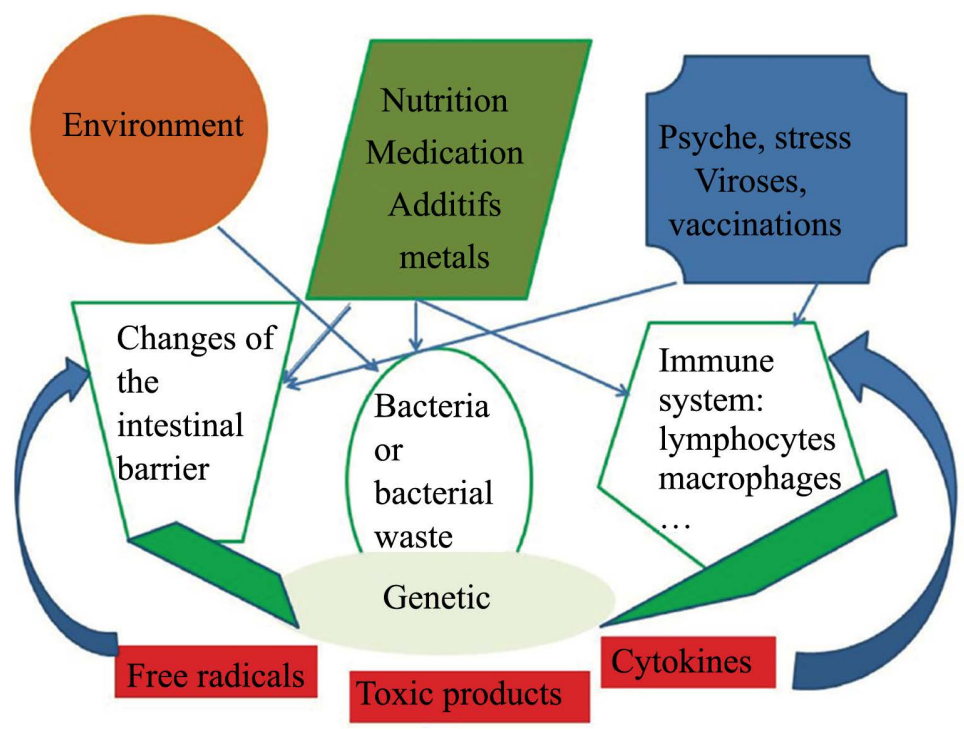

Figure 1. Interactions between external and internal factors, including genetic factors that influence the homeostasis. 


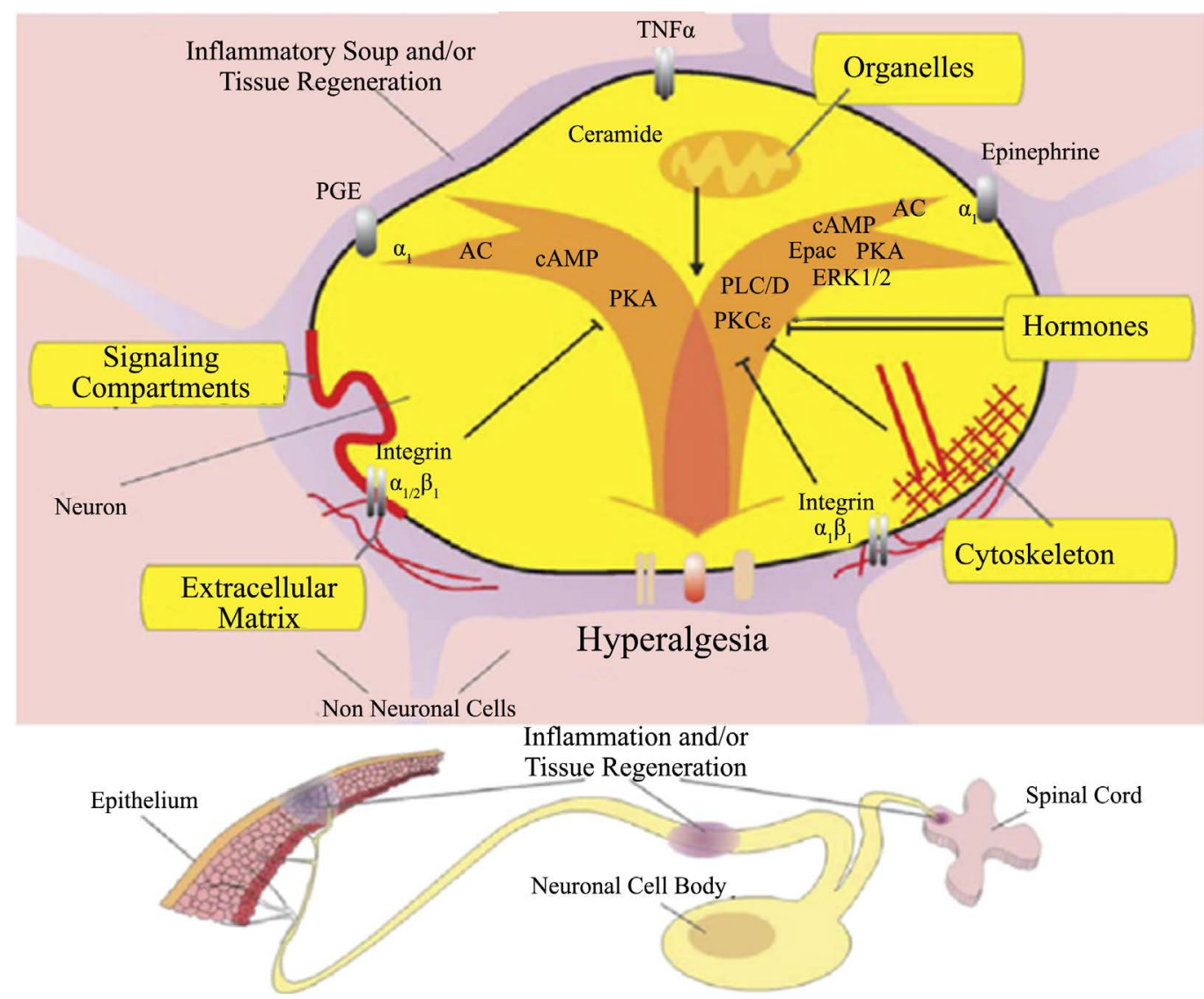

Figure 2. Hyperalgesia at cellular level. The sensibilization involves the ion channels that control the electrophysiologic properties of the cell. The organelles, the subcellular compartimentation, the secondary signalization, the micro-environment all participate in the state of sensibilization [8].

system and the brain, which appear to play a prominent role in nociception. Glial cells, also known as neuroglia, arenon-conducting cells that modulate neurotransmission at the synaptic level.

Solving the problem will require focus on biological mechanisms and strategic thinking

\subsection{Acupuncture How Does It Work}

All types of acupuncture make lesions in the soft tissue that activate the survival mechanisms that normalize homeostasis and promote healing. The needling is perceived as a foreign invader and the needle-induced lesion as an injury of mechanical trauma to the tissues. It may cause tiny bleedings in the soft tissues and thus induce hemodynamic changes such as dilation of the arterioles and the opening of new capillaries and venular beds in the area.

Needles inserted in injured and inflamed tissues trigger a tiny, local anti-inflammatory reaction that speeds up the inflammatory process. It is a protective strategy of the body, when it last too long it becomes, however, disabling.

Centrally the needle-induced lesions stimulate the survival systems in the brain such as the nervous, endocrine, immune and cardiovascular systems and normalize the physiological activities of the whole body.

The improvement of the knowledge on the biological effects of acupuncture equally highlights its complexity.

The initial healing phase of acupuncture can be explained by the mechanotransduction (see Figure 3). The transductional response of the connective tissue cell consists of a multitude of potential reaction and regulations that induce target proteins.

Peripherally the lesions trigger physiological reactions around the needling site, a cascade of survival reactions is initiated. Calcitonin Gene Related Peptide (CFRP) and nitrous oxide (NO) are released via the reflex of the axon due to the increased blood flow in the muscle after manual acupuncture and contribute to the tissue 


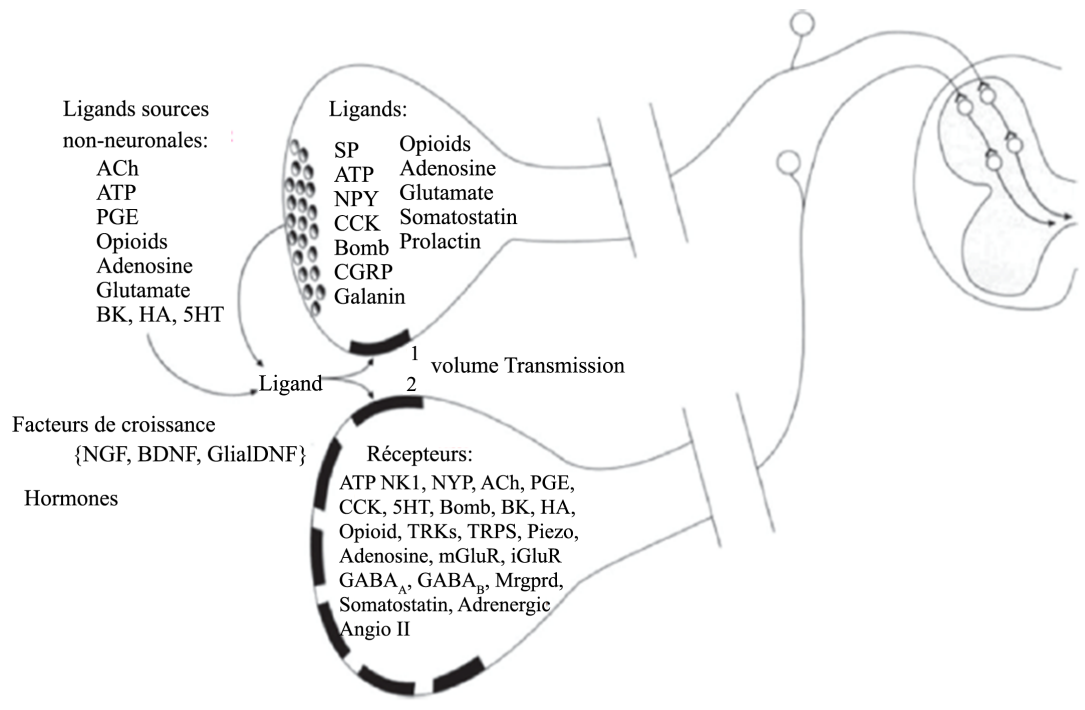

...One can begin to imagine that each terminal resembles to a tiny brain! The complexity of nociceptors has led us to think of them as having a 'mind of their own'.

Figure 3. Interactions at the nerve endings [13] with permission of the publisher.

healing [11] [12].

This makes acupuncture a physiological therapy whereby the brain responds to the stimulation of peripheral sensory nerves by the needles. Acupuncture does not treat a particular symptom but it normalizes the physiological homeostasis and promotes self-healing.

Molecular biology showed that primary nociceptors are complex in the ligand expression of neurotransmitters and receptors, enabling rich autocrine and paracrine interactions.

Primary nociceptors can modify (facilitation or inhibition) sensorial information even before transmission to the central nervous system.

They generate efferent messages towards the innervated tissues (bidirectional communication).

Nociceptors seem to have a mind of their own [13] (see Figure 3).

Animal experiments in neuropathic pain showed that pain control may be effected by modulation of different molecules and receptors such as GABA, glutamate and its different receptors (AMPA, NMDA ...), the serotonin (5HT) system, the opioid system, norepinephrine (NE), cholinergic receptors etc. Note that on neuropathic pain, electro acupuncture (EA) at low frequency $(2 \mathrm{~Hz})$ has analgesic effect much higher than high frequency (100 $\mathrm{Hz})$.

Functional neuroimaging studies, documented brain responses in cortical and subcortical regions, including activation in the sensorimotor cortical and deactivation in the limbic-para limbic-neocortical network with acupuncture treatment. These brain areas are associated with a pain matrix responsible for modulating the sensation of pain and affective pain perception [14].

The therapeutic response to acupuncture such as down regulation of inflammation and autonomic nervous system mediated pain relief was postulated to be provided by a mechanistic pathway of the autonomic nervous system.

Napadow et al. [15] were the first to apply event related (er)-fMRI to evaluate the brain correlates of acupuncture stimulation, which appears to be associated with different autonomic nervous system outflow responses to needle stimuli and may result from different sensations elicited by stimuli at different bodily locations.

Harris et al. [14] compared short- and long-term effects of traditional Chinese acupuncture versus sham acupuncture treatment on in vivo $\mu$-opioid receptor (MOR) binding availability in chronic pain patients diagnosed with fibromyalgia. Positron emission tomography (PET) was performed once during the first treatment session 
and then repeated a month later following the eighth treatment. Acupuncture therapy evoked short-term increases in MOR binding potential, in multiple pains and sensory processing regions including the cingulate (dorsal and subgenual), insula, caudate, thalamus, and amygdala. Acupuncture therapy also evoked long-term increases in MOR binding potential in some of the same structures including the cingulate (dorsal and perigenual), caudate, and amygdala. These short- and long-term effects were absent in the sham group where small reductions were observed, an effect more consistent with previous placebo PET studies.

\section{Integrative Neuromuscular Acupoint System (INMAS)}

The integration of Western and traditional Oriental medicine is a working model for pain management. It applies the modern biomedical principles and retains the major principles of oriental medicine: restoration of the physiological homeostasis, maximizing the self-healing without side effects and treats both the cause and the symptoms of the disease.

INMAS is simple, reproducible and predictable.

The integrative method applies the modern biomedical principles but also maintains the principles of Oriental medicine.

1) Restoring physiological homeostasis (the balance of yin and yang)

2) Maximizing self-healing without side-effects

3) Treating both ben (the root of the disease, the whole body) and biao (the symptoms of the disease)

INMAS has the characteristics required by a clinical procedure:

a) Simplicity: the whole procedure from evaluation of the patient to insertion of needles can be performed in a very short time in the clinic

b) Reproducibility: all the procedures and therapeutic results are reliable and reproducible by any practitioner.

c) Predictability: this method enable the practitioner to predict the results of the treatment as follows:

(1) Whether the patient will respond to acupuncture treatment or not

(2) If the patient is a responder, how many treatments will be needed to achieve pain relief

(3) Whether the pain will return at some time after the initial relief is achieved [16].

\section{Auriculotherapy}

The scientific basis of auriculotherapy goes back to 3500 BC. It was first performed in an empiric way. It was rediscovered in the 1950's by Paul Nogier and officially recognized by the World Health Organization (WHO). This discipline was introduced in modern medicine due to the neurosciences and the functional neuroimaging (fMRI)

The effect of auriculotherapy can be explained by neuro embryologic and neurogenetic mechanisms. During the embryonic and fetal development a map identical to the link between body and brain would be imprinted on the ear [17] (see Figure 4).

Auriculotherapy is an adjunct to traditional acupuncture and over the last 60 years has developed into a distinct treatment system of its own. It is based on a set of anatomical maps superimposed onto the ear. Stimulating a point on the map is proposed to affect the gross anatomical organ associated with that point.

\section{The Evidence for Acupuncture Treatment of Pain}

Acupuncture was shown to be beneficial in a variety of painful conditions such as osteoarthritis of the knee [18]-[20], chronic mechanical neck pain [21], Bell palsy [22], and symptoms associated with fibromyalgia [23]. It was shown to be superior to physical therapy and usual care in patients with chronic low back pain [24]-[26] and proved to be cost effective [27]. Conclusions from a meta-analysis show that acupuncture is an effective treatment for chronic pain [28].

Acupuncture treatments reduced symptoms of chronic prostatitis/chronic pelvic pain [29].

Acupuncture is being used in the palliative care of cancer to alleviate pain [30] [31], dysfunction [31] fatigue [32] [33].

Early studies of auriculotherapy have demonstrated beneficial effects on both pain and anxiety including pain associated with cancer, [30] knee arthroscopy, [34] and hip fracture and hip arthroplasty [35]. Several recent small studies have suggested that auricular acupuncture alone can relieve pain and anxiety in the pre-hospital 


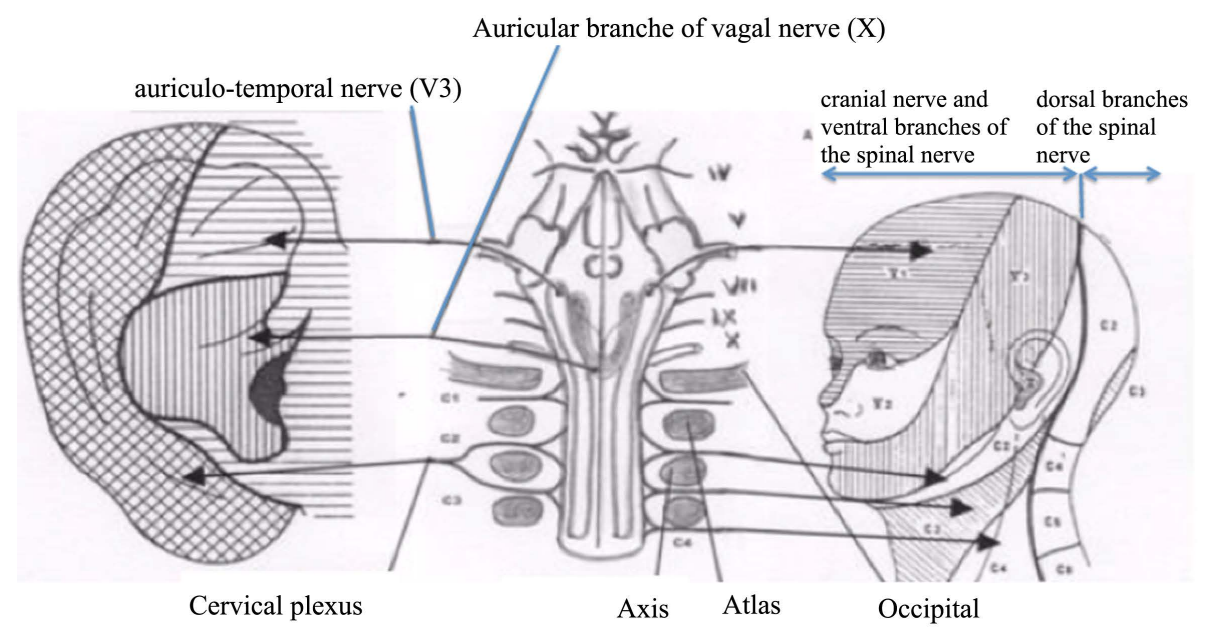

Figure 4. Facial and auricular afferents of the spinal nerve (adapted from INSERM 2013).

transport phase of hip fracture [35] [36] and reduce acute pain due to a variety of causes in the emergency department setting [37].

A recent systematic review and meta-analysis included 17 studies on acute pain, postoperative pain and chronic pain. The evidence from this review suggests that auriculotherapy may be effective for the treatment of a variety of types of pain, especially postoperative pain, and may be a reasonable adjunct for patients having difficulties with pharmacologic pain therapies [38] (see Diagram 1).

In the field of acupuncture research there is an implicit yet unexplored assumption that the evidence on manual and electrical stimulation techniques, derived from basic science studies, clinical trials, systematic reviews, and meta-analyses, is generally interchangeable. Such interchangeability would justify a bidirectional approach to acupuncture research, where basic science studies and clinical trials each inform the other.

The synergy of acupuncture in an integrative process with pharmaconutritional close to pharmacological treatment can improve the clinical picture and promote the revalidation.

Genetic variations may modulate the answer to treatment, but does not explain completely the variability of the therapeutic efficiency, which exists between individuals.

Pain is a complex matrix that is intimately intertwined with expectation. Acupuncture clearly affects this matrix in both specific and non-specific manner that is consistent with its specific clinical effects, as well as the effects of expectation on pain relief [39].

\section{Our Experience}

These considerations regarding the physiology, neuroendocrinology, peripheral and central sensitization, epigenetic factors such as nutrition and intestinal flora, social, psychological and affective were for over 25 years integrated in the evaluation at the pain clinic Chirec, this according to the bio psychosocial model to implement a multimodal treatment.

The evaluation focuses on the predisposing and promoting factors for chronicity and therapeutic failure.

These multiple factors are articulated in the biological, neurophysiologic, peripheral and central or the inflammation cascade that induces alterations in the cellular homeostasis and plasticity of the nervous system.

Why the focus on nutrition? Specific nutritional deficiencies can be associated with pain states. For example, a Mayo Clinic study discovered that pain patients with insufficient levels of vitamin D were taking twice the amount of opioids for twice as long as patients without a deficiency. The balance of microorganisms can determine health or disease by affecting the absorption of nutrients .

Processed foods and drugs such as proton pump inhibitors, NSAIDs, antibiotics, steroids, and hormones can adversely affect the microbiome [40].

The role of acupuncture in this multidisciplinary process is a pertinent physiological regulator. The therapeutic and post effect are clearly augmented by the cellular physiological rehabilitation, of which we described certain steps. 


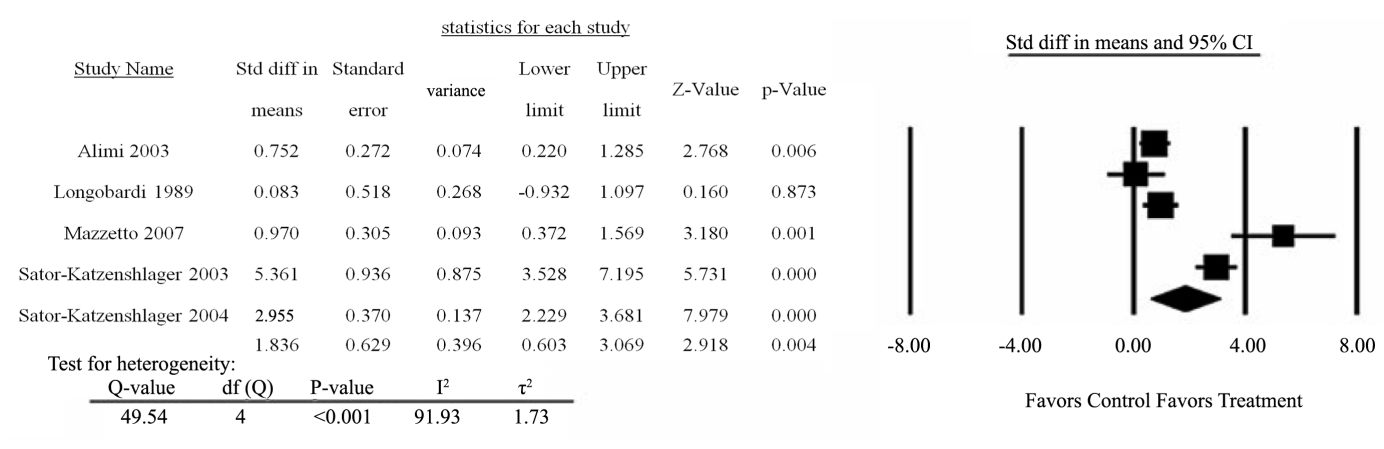

Diagram 1. Auriculotherapy for chronic pain versus control: Pain score (reprinted with permission of the publisher).

This illustrates the interest of the acupuncture stimulus and its interaction with primary nociceptors that have complex regulating capacities.

During the diagnostic steps the reasoning of the traditional Chinese medicine is integrated with the chronobiology purpose and the epigenetics factors involved, thus allowing a strategy for acupuncture that addresses the chronicity and the modified parameters such as sleep, nutrition, reactive depression and the different vicious circles induced by chronic pain (peripheral and central sensitization).

Scientific auricular acupuncture and its neurophysiologic contribution are complementary to other treatment options and induce a prolonged and reproducible effect.

\section{Case Reports}

Personally we obtained the best results using an integrative approach as illustrated in the case report below.

- A 45-year-old woman with confirmed type 3 EDS suffered extreme pain, fatigue and sleep disturbances, not controlled by pharmacological analgesics [41]. The disease has progressed over 22 years pain was never controlled. The patient has undergone several arthroplasties and has total alopecia, which has led to depression and suicidal attempts.

Multidisciplinary biopsychosocial evaluation, cognitive and systemic, acupuncture treatment close to the pharmaco-nutritional strategy focused on liver and gastrointestinal mucosa, were performed.

3 days after the 1st acupuncture session, 50\% pain reduction was observed and analgesic use was progressively reduced. Family problems leading to emotional stress temporarily increased the pain level.

After 4 acupuncture sessions pain could be managed with low dose of analgesics on demand. Sleep recovered and the patient reported $50 \%$ decrease in fatigue. Hair has grown $4 \mathrm{~cm}$.

Six months later, monthly treatment controlled pain with few analgesics on demand. Pharmaco-nutrition-nalstrategies focused on methylation, intestinal mucosa, joints and muscles are associated (Figure 5).

- We also have noticed favorable results in patients suffering post-surgical thoracic pain.

The first patient is a female referred to the pain clinic with refractory thoracic pain after lung resection for cancer. She suffers constant burning pain superimposed by episodes of electrical discharges and tingling. Pharmacological and non-pharmacological treatment proved to be ineffective in reducing pain intensity. The pain interferes with the sleep and has a negative impact on the general status. The pain intensity is estimated to be $8 / 10$ on a visual analogue scale. Clinical examination showed a hypoesthesia to touch and pinprick from Th4 to Th7. The scar is red and inflamed without a zone of local hyperesthesia. There is an important thoracic kyphosis and paravertebral muscle tensions. The DN4 (neuropathic pain score) is 6/10, suggestive for neuropathic pain. The patient lives on her own and show signs of moderate depression.

The patient was treated with auriculoterapy on the peripheral and central projection zones of pain and a serotonin point.

Evolution VAS 4/10 after the first session and $0 / 10$ at the second consultation

The second patient is a 50-year old male who consults for thoracic pain post thoracotomy that was performed 2 year ago for a coronary bypass [42]. Pain initially improved but returned after the death of his spouse. He describes thoracic oppressions, a hypersensitivity of sternal scar, paresthesia around the scar zone and diffuse pain in the thorax upon mobilization. Friction of the clothing is intolerable. Pain, fear and insomnia induce anorexia. 


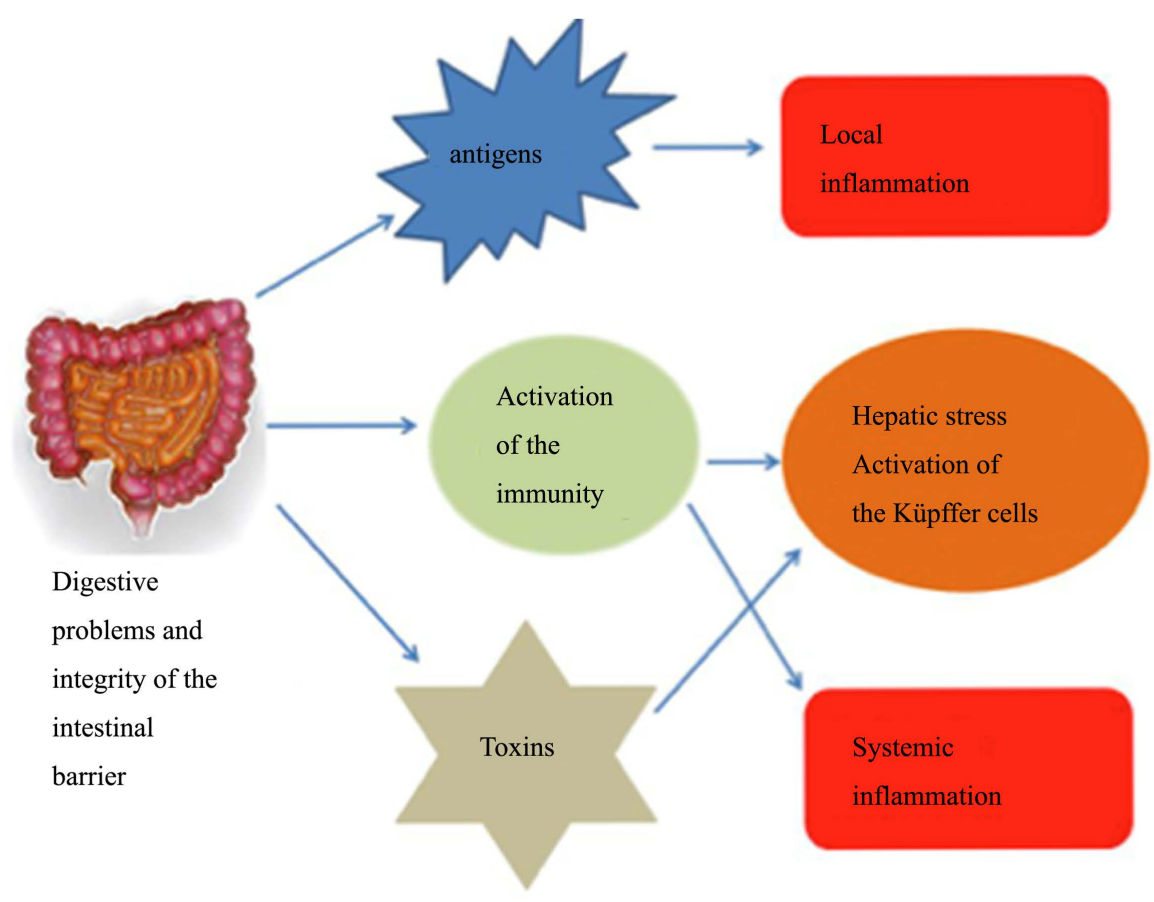

Figure 5. Link intestine-liver-inflammation.

This pain has a nociceptive and neuropathic mechanism. Acupuncture was performed sympathetic and sensitive metamer, reticular point and thalamus point.

Evolution, with a monthly and trimestral treatment the pain was controlled.

Some stressful situation induced recurrence showing the plasticity of the central brain and the memory vicious circle.

- Central Pain Syndromes (CPS) include for instance post-stroke syndrome [43]. Patients experience severe pain, sensory abnormalities, and emotional distress. Intolerable side effects hinder pharmacological pain control

We report the multimodal approach of a refractory central pain post stroke patient with acupuncture and the natural compound palmitoylethanolamide (PEA), focusing on chronobiology and epigenetic factors involved in drug response and side effects.

A 37-year female patient suffered stroke with thalamic pain and discomfort in the right body half. Pharmacological treatment was insufficient. After two years left sided motor cortex stimulation was performed. After 16 years the patient attended the pain clinic with severe refractory pain, major drug side effects, distress, obesity, sleep disorders, and no social life (VAS 9/10).

Treatment consisted of methadone $120 \mathrm{mg}$ and duloxetine $120 \mathrm{mg}$. Acupuncture resulted in instable pain reduction. We added PEA, vitamin D and nutritional rehabilitation. Pain stabilized (VAS 4/10) with better response to the same dose methadone. The patient recovered sleep, lost weight, improved daily activity, social contacts, and took the first vacation since 20 years.

- Acute and chronic pain, initiate a variety of responses that modify the general homeostasis. A multidisciplinary biopsychosocial evaluation allows to identify the associated factors and what elements to improve this physiological status. A strategy that includes integrated phamaco nutritionnal and acupuncture approach close to analgesics shows a revalidation of the general status.

As described in literature, we also observed a better response and tolerance of the treatment, a dose reduction and a significant improvement of the parameters involved in the chronification.

\section{Conclusions}

Acupuncture is effective for the treatment of chronic pain and is therefore a reasonable referral option. Significant differences between true and sham acupuncture indicate that acupuncture is more than a placebo. However, 


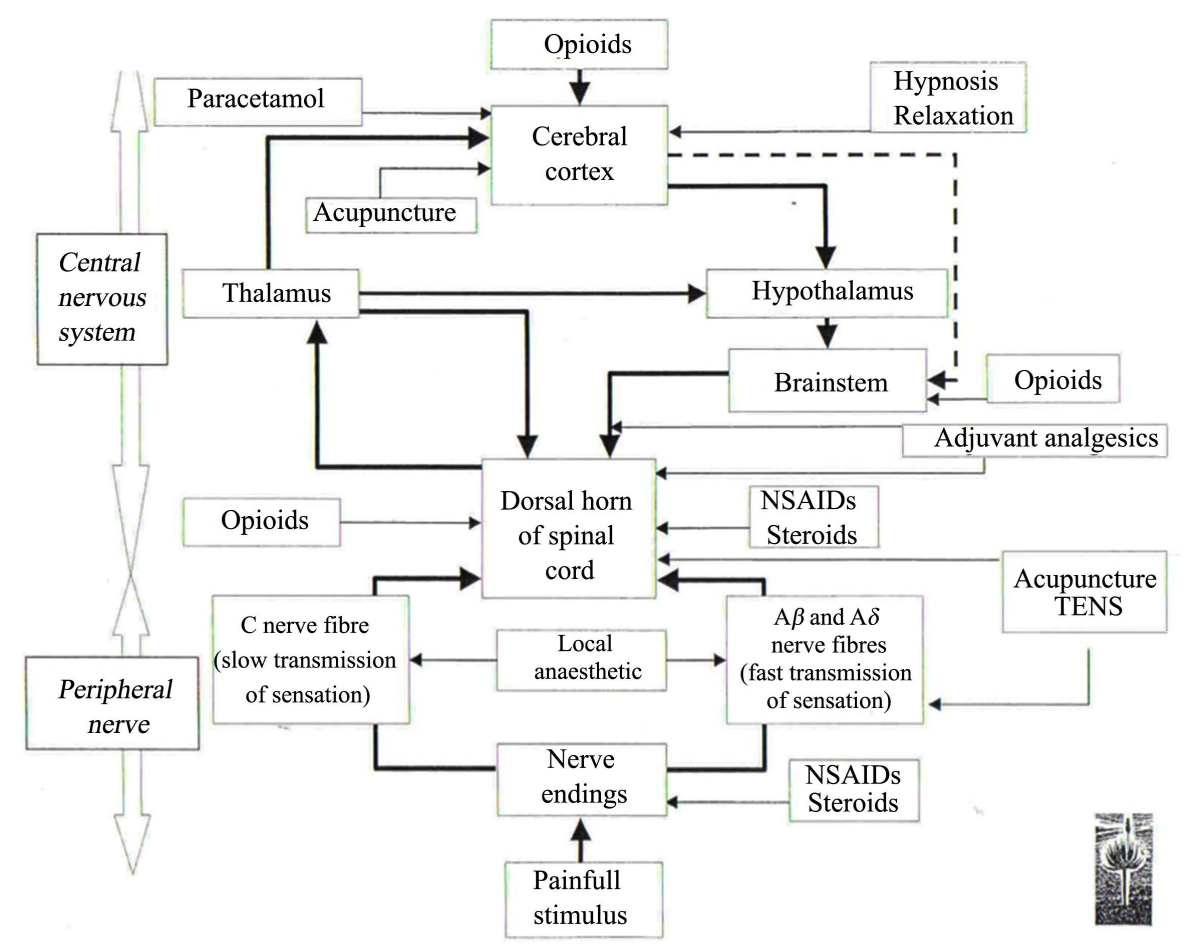

Figure 6. Pain treatment with acupuncture synergy.

these differences are relatively modest, suggesting that factors in addition to the specific effects of needling are important contributors to the therapeutic effects of acupuncture.

"A perfect knowledge of the cellular biochemistry, means staying close to the patients. A mitochondrial nutritional environment is a prerequisite for health protection and the guarantee for a harmonious aging.” [44].

Both principles of classic acupuncture and the latest scientific explanation of the underlying mechanisms explained above show that acupuncture involves central and peripheral mechanisms. When treating soft tissue pain, needling lesions are made directly into the painful tissue. These local lesions activate neuroendocrine immunologic and cardiovascular reactions around the needling site. This local needling reaction directly disseminates into the painful nerves and induces repair of the damaged soft tissues.

The Chinese have an old saying: "That nine on ten diseases produce pain and according to statistics $85 \%$ of the pain is soft tissue pain." [45].

Clinical evidence shows that acupuncture is effective for a variety of health problem, but particularly for cases in which Western medicine has little to offer, especially in pain management. It is easy to administer and has no side effects if performed by a trained practitioner. Acupuncture transforms the non-specific effect of needing into a specific effect for specific symptoms by creating, or inoculating the lesions directly in the painful areas.

Including acupuncture in a multimodal treatment program is in-line with the current research to enhance efficacy when combining drugs from different classes, close to pharmaconutritional treatment.

A thorough evaluation of the different components of the pain problem, allows selecting a multimodal treatment schedule with acupuncture.

It is now a well-established fact that these combinations may lead to synergies, enabling the physician to treat patients with different drugs, improve efficacy, while reducing side effects, and drugs can be administered in lower dosages (see Figure 6).

\section{References}

[1] Ernst, E., Lee, M.S. and Choi, T.Y. (2011) Acupuncture: Does It Alleviate Pain and Are There Serious Risks? A Review of Reviews. Pain, 152, 755-764. http://dx.doi.org/10.1016/j.pain.2010.11.004

[2] Han, J.S. (2003) Acupuncture: Neuropeptide Release Produced by Electrical Stimulation of Different Frequencies. Trends in Neurosciences, 26, 17-22. http://dx.doi.org/10.1016/S0166-2236(02)00006-1 
[3] Liu, B., et al. (2012) Altered Small-World Efficiency of Brain Functional Networks in Acupuncture at ST36: A Functional MRI Study. PLoS One, 7, e39342. http://dx.doi.org/10.1371/journal.pone.0039342

[4] Zeng, F., et al. (2008) Application of PET-CT Technique to the Research on Central Mechanism of Acupuncture Effects. Zhen Ci Yan Jiu, 33, 284-286.

[5] Dhond, R.P., Kettner, N. and Napadow, V. (2007) Neuroimaging Acupuncture Effects in the Human Brain. Journal of Alternative and Complementary Medicine, 13, 603-616. http://dx.doi.org/10.1089/acm.2007.7040

[6] Junien, C. (2008) Nutrition and Epigenetic. INRA. INSERN U 781.

[7] Mazic-de Sonis, A. (2014) Multimodal Management of Chronic Pain: Litterature on the Basic Research and Clinical Expertise. In: 14 Congrès national de la SFETD, Toulouse.

[8] Plaghki, L. (2014) Update on Nociceptors. In: GRID, Ed., Quarterly Meeting, October 2014, Liège.

[9] Mazic-de Sonis, A. (2014) Neuroscience and Acupuncture Understanding the Mechanisms of Action: A Summary of the Cerebral Imaging Research. In: SFETD, Ed., 14 Congrès national de la SFETD, Toulouse.

[10] Delmas, P. (2012) Sodium Channels and Pain. Douleurs et Analgésie, 13, A34-A35.

[11] Sandberg, M., et al. (2005) Different Patterns of Blood Flow Response in the Trapezius Muscle Following Needle Stimulation (Acupuncture) between Healthy Subjects and Patients with Fibromyalgia and Work-Related Trapezius Myalgia. European Journal of Pain, 9, 497-510. http://dx.doi.org/10.1016/j.ejpain.2004.11.002

[12] Shinbara, H., et al. (2013) Participation of Calcitonin Gene Related Peptide Released via Axon Reflex in the Local Increase in Muscle Blood Flow Following Manual Acupuncture. Acupuncture in Medicine, 31, 81-87. http://dx.doi.org/10.1136/acupmed-2012-010253

[13] Carlton, S.M. (2014) Nociceptive Primary Afferents: They Have a Mind of Their Own. The Journal of Physiology, 592, 3403-3411. http://dx.doi.org/10.1113/jphysiol.2013.269654

[14] Harris, R.E., et al. (2009) Traditional Chinese Acupuncture and Placebo (Sham) Acupuncture Are Differentiated by Their Effects on Mu-Opioid Receptors (MORs). Neuroimage, 47, 1077-1085. http://dx.doi.org/10.1016/j.neuroimage.2009.05.083

[15] Napadow, V., et al. (2013) Brain Correlates of Phasic Autonomic Response to Acupuncture Stimulation: An EventRelated fMRI Study. Human Brain Mapping, 34, 2592-2606. http://dx.doi.org/10.1002/hbm.22091

[16] Ma, Y.-T., Ma, M. and Hee, C.Z. (2004) Introduction to the Practical Application of the Integrative Neuromuscular Acupoint System in Biomedical Acupuncture for Pain Management: An Integrative Approach. Elsevier, Churchil Livingstone, St. Louis, 104-107.

[17] Gueguen, J., et al. (2013) Evaluation de l'efficacité de la pratique de l'auriculothérapie. INSERM U669.

[18] Berman, B.M., et al. (2004) Effectiveness of Acupuncture as Adjunctive Therapy in Osteoarthritis of the Knee: A Randomized, Controlled Trial. Annals of Internal Medicine, 141, 901-910. http://dx.doi.org/10.7326/0003-4819-141-12-200412210-00006

[19] Scharf, H.P., et al. (2006) Acupuncture and Knee Osteoarthritis: A Three-Armed Randomized Trial. Annals of Internal Medicine, 145, 12-20. http://dx.doi.org/10.7326/0003-4819-145-1-200607040-00005

[20] Witt, C.M., et al. (2006) Acupuncture in Patients with Osteoarthritis of the Knee or Hip: A Randomized, Controlled Trial with an Additional Nonrandomized Arm. Arthritis \& Rheumatology, 54, 3485-3493. http://dx.doi.org/10.1002/art.22154

[21] White, P., et al. (2004) Acupuncture versus Placebo for the Treatment of Chronic Mechanical Neck Pain: A Randomized, Controlled Trial. Annals of Internal Medicine, 141, 911-919. http://dx.doi.org/10.7326/0003-4819-141-12-200412210-00007

[22] Xu, S.B., et al. (2013) Effectiveness of Strengthened Stimulation during Acupuncture for the Treatment of Bell Palsy: A Randomized Controlled Trial. Canadian Medical Association Journal, 185, 473-479. http://dx.doi.org/10.1503/cmaj.121108

[23] Martin, D.P., et al. (2006) Improvement in Fibromyalgia Symptoms with Acupuncture: Results of a Randomized Controlled Trial. Mayo Clinic Proceedings, 81, 749-757. http://dx.doi.org/10.4065/81.6.749

[24] Hsieh, L.L., et al. (2006) Treatment of Low Back Pain by Acupressure and Physical Therapy: Randomised Controlled Trial. British Medical Journal, 332, 696-700. http://dx.doi.org/10.1136/bmj.38744.672616.AE

[25] Thomas, K.J., et al. (2006) Randomised Controlled Trial of a Short Course of Traditional Acupuncture Compared with Usual Care for Persistent Non-Specific Low Back Pain. British Medical Journal, 333, 623. http://dx.doi.org/10.1136/bmj.38878.907361.7C

[26] Haake, M., et al. (2007) German Acupuncture Trials (GERAC) for Chronic Low Back Pain: Randomized, Multicenter, Blinded, Parallel-Group Trial with 3 Groups. Archives of Internal Medicine, 167, 1892-1898. 
http://dx.doi.org/10.1001/Archinte.167.17.1892

[27] Ratcliffe, J., et al. (2006) A Randomised Controlled Trial of Acupuncture Care for Persistent Low Back Pain: Cost Effectiveness Analysis. British Medical Journal, 333, 626. http://dx.doi.org/10.1136/bmj.38932.806134.7C

[28] Vickers, A.J., et al. (2012) Acupuncture for Chronic Pain: Individual Patient Data Meta-Analysis. Archives of Internal Medicine, 172, 1444-1453. http://dx.doi.org/10.1001/archinternmed.2012.3654

[29] Lee, S.W., et al. (2008) Acupuncture versus Sham Acupuncture for Chronic Prostatitis/Chronic Pelvic Pain. American Journal of Medicine, 121, 79.e1-e7.

[30] Alimi, D., et al. (2003) Analgesic Effect of Auricular Acupuncture for Cancer Pain: A Randomized, Blinded, Controlled Trial. Journal of Clinical Oncology, 21, 4120-4126. http://dx.doi.org/10.1200/JCO.2003.09.011

[31] Pfister, D.G., et al. (2010) Acupuncture for Pain and Dysfunction after Neck Dissection: Results of a Randomized Controlled Trial. Journal of Clinical Oncology, 28, 2565-2570. http://dx.doi.org/10.1200/JCO.2009.26.9860

[32] Vickers, A.J., et al. (2004) Acupuncture for Postchemotherapy Fatigue: A Phase II Study. Journal of Clinical Oncology, 22,1731-1735. http://dx.doi.org/10.1200/JCO.2004.04.102

[33] Molassiotis, A., et al. (2012) Acupuncture for Cancer-Related Fatigue in Patients with Breast Cancer: A Pragmatic Randomized Controlled Trial. Journal of Clinical Oncology, 30, 4470-4476. http://dx.doi.org/10.1200/JCO.2012.41.6222

[34] Usichenko, T.I., et al. (2007) Auricular Acupuncture for Pain Relief after Ambulatory Knee Surgery: A Randomized Trial. Canadian Medical Association Journal, 176, 179-183. http://dx.doi.org/10.1503/cmaj.060875

[35] Usichenko, T.I., et al. (2005) Auricular Acupuncture for Pain Relief after Total Hip Arthroplasty: A Randomized Controlled Study. Pain, 114, 320-327. http://dx.doi.org/10.1016/j.pain.2004.08.021

[36] Barker, R., et al. (2006) Out-of-Hospital Auricular Acupressure in Elder Patients with Hip Fracture: A Randomized Double-Blinded Trial. Academic Emergency Medicine, 13, 19-23. http://dx.doi.org/10.1111/j.1553-2712.2006.tb00979.x

[37] Goertz, C.M., et al. (2006) Auricular Acupuncture in the Treatment of Acute Pain Syndromes: A Pilot Study. Military Medicine, 171, 1010-1014. http://dx.doi.org/10.7205/MILMED.171.10.1010

[38] Asher, G.N., et al. (2010) Auriculotherapy for Pain Management: A Systematic Review and Meta-Analysis of Randomized Controlled Trials. Journal of Alternative and Complementary Medicine, 16, 1097-1108. http://dx.doi.org/10.1089/acm.2009.0451

[39] Lewith, G.T., White, P.J. and Pariente, J. (2005) Investigating Acupuncture Using Brain Imaging Techniques: The Current State of Play. Evidence-Based Complementary and Alternative Medicine, 2, 315-319. http://dx.doi.org/10.1093/ecam/neh110

[40] IASP (2014) IASP Pain: Clinical Update.

[41] Mazic-de Sonis, A. (2013) Multimodal Management of Pain in a Patient with Ehlers Danlos Syndrome. EFIC, Firenze.

[42] Mazic-de Sonis, A. (2010) Management of Post Thoracotomy Pain in ABMA Congress. ABMA, Brussels.

[43] Mazic-de Sonis, A. (2015) Multimodal Stepped Care Approach Whith Acupuncture and Palmitoylethanolamide for Post Stroke Pain in XXII World Congress of Neurology. WCN, Santiago.

[44] Castronovo, V. (2010) Medecine nutritionelle et fonctionelle. Cours de formation, Liège.

[45] White, A., Cummings, M. and Filshie, J. (2008) Summary of the Medicinal Occidental Acupuncture. Elsevier Masson, Issy-les-Moulineaux cedex. 